\title{
Risk stratification of patients with nodal involvement in upper tract urothelial carcinoma: value of lymph-node density
}

Christian Bolenz, Shahrokh F. Shariat ${ }^{1}$, Mario I. Fernández ${ }^{2}$, Vitaly Margulis ${ }^{3}$, Yair Lotan ${ }^{1}$, Pierre Karakiewicz ${ }^{4}$, Mesut Remzi ${ }^{5}$, Eiji Kikuchi ${ }^{6}$, Richard Zigeuner ${ }^{7}$, Alon Weizer ${ }^{8}$, Francesco Montorsi ${ }^{9}$, Karim Bensalah ${ }^{10}$, Christopher G. Wood ${ }^{3}$, Marco Roscigno ${ }^{9}$, Cord Langner ${ }^{7}$, Theresa M. Koppie ${ }^{11}$, Jay D. Raman ${ }^{12}$, Shuji Mikami ${ }^{6}$, Maurice Stephan Michel and Philipp Ströbel Mannheim Medical Center, University of Heidelberg, Germany, 'University of Texas Southwestern Medical Center, Dallas, TX, USA (currently, SFS is at Memorial Sloan-Kettering Cancer Center, New York, NY, USA), ${ }^{2}$ linica Alemana de Santiago, Chile, ${ }^{3}$ University of Texas MD Anderson Cancer Center, Houston, TX, USA, ${ }^{4}$ University of Montreal, Montreal, Quebec, Canada, ${ }^{5}$ Medical University of Vienna, Vienna, Austria, ${ }^{6}$ Keio University School of Medicine, Tokyo, Japan, ${ }^{7}$ Medical University Graz, Graz, Austria, ${ }^{8}$ University of Michigan, Ann Arbor, MI, USA, ${ }^{9}$ Vita-Salute University, Milan, Italy, ${ }^{10}$ University of Rennes, Rennes, France, "University of California Davis, Sacramento, CA, and ${ }^{12}$ Penn State Milton S. Hershey Medical Center, PA, USA

Accepted for publication 18 June 2008

$\mathrm{CB}$ and SFS contributed equally to this paper

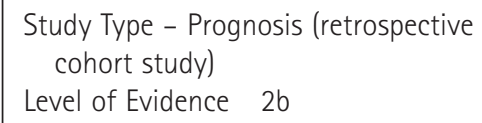

OBJECTIVE

To determine the risk factors associated with clinical outcome in patients with lymph node $(\mathrm{LN})$-positive urothelial carcinoma of the upper urinary tract (UTUC) treated with radical nephroureterectomy (RNU) and lymphadenectomy, focusing on the concept of LN density (LND).

\section{PATIENTS AND METHODS}

Patients undergoing RNU with regional lymphadenectomy were identified through multi-institutional databases. All pathology slides were re-evaluated by genitourinary pathologists unaware of the clinical data. The exposure variable used was LND (continuously coded and that of all possible thresholds) with recurrence-free and disease-specific survival (DSS) serving as the outcome measures.

\section{RESULTS}

Of 432 patients undergoing RNU with lymphadenectomy, 135 (31\%) had LN metastases. Within a median follow-up of 4.1 years, 90 of the 135 patients with LN metastases $(68 \%)$ had disease recurrence and 76 (58\%) died from UTUC. The mean (SEM) 5-year recurrence-free and DSS probabilities were 27 (4)\% and 33 (5)\%, respectively. The median (range) LND was 50 (3-100)\%. The most informative threshold for LND in relation to outcome was 30\%. In multivariable analyses that adjusted for the effects of tumour stage and grade, patients with a LND of $\geq 30 \%$ were at greater risk of both cancer recurrence, with 5-year rates of 25 (5)\% vs 38 (8)\% (hazard ratio 1.8,
$P=0.021)$ and mortality, with 5-year rates of 30 (6)\% vs 48 (9)\% (1.7, $P=0.032)$ compared to those with a LND of $<30 \%$. Our results are primarily limited by a lack of standardization in the lymphadenectomy template.

\section{CONCLUSION}

We evaluated the concept of LND for the first time in UTUC. LND provides additional prognostic information in patients with node-positive disease after RNU. The use of LND in clinical trials might provide an additional insight into the value of LN dissection in patients undergoing RNU.

\section{KEYWORDS}

urothelial carcinoma, urinary tract cancer, lymphatic metastases, lymph node dissection, prognosis

\section{INTRODUCTION}

Radical nephroureterectomy (RNU) with removal of a bladder cuff is the standard and most effective treatment for patients with invasive upper tract urothelial carcinoma (UTUC) [1-3]. Of patients with
UTUC, 20-40\% have locally advanced disease with lymph node (LN) metastases at the time of diagnosis [4-6], with a 5-year diseasespecific survival (DSS) probability reported as low as $12 \%$ [7]. Both in LN-positive UTUC and bladder UC, adjuvant treatment strategies after surgery, e.g. chemotherapy or radiotherapy, have been used in an attempt to improve survival $[8,9]$.

To date, there are no data on risk stratification of patients undergoing RNU with lymphadenectomy for LN-positive UTUC. Lymphadenectomy allows accurate tumour 
staging and there is recent evidence of therapeutic effects in both patients with or with no LN metastases $[4,10]$. In bladder UC, radical cystectomy with lymphadenectomy alone might cure $5-34 \%$ of patients with microscopic LN-positive disease [11]. Extended lymphadenectomy also adds significant therapeutic benefit in both LNnegative and -positive bladder UC [12-14]. For UTUC it was also recently reported that extended lymphadenectomy in addition to RNU provides a survival benefit in patients with locally advanced UTUC $[4-6,15]$.

Despite the evidence of diagnostic and potential therapeutic benefits of $L N$ dissection for UC, lymphadenectomy is used sporadically and haphazardly for UTUC. Absolute numbers of LNs and number of positive LNs reported in most series fail to capture the disease burden in the patient and the extent of dissection by the surgeon. LN density (LND) (the percentage of the number of LNs involved with tumour divided by the total number of LNs examined) has been evaluated in bladder UC [14]. Several studies suggest that the LND might have greater prognostic value than absolute numbers of involved LNs because it takes into account the potential therapeutic value of an extended LN dissection $[14,16,17]$. We evaluated the effect of LND on recurrence-free and DSS in a multi-institutional retrospective cohort of patients undergoing RNU with regional lymphadenectomy.

\section{PATIENTS AND METHODS}

Institutional review board approval was obtained by all 12 study sites in accordance with local regulations. A computerized databank was generated for data transfer (UT Southwestern Medical Center, Dallas, Texas). Data integrity was insured during integration and initial data analysis by resolving data inconsistency with the local study sites. Before the final analysis, the database was 'frozen' from any additional modifications and the final dataset was produced for the current analysis.

Of 1363 patients who had RNU between 1992 and 2006, 432 (31.7\%) were identified who had a lymphadenectomy with removal of at least one LN involved with tumour in addition to RNU. The present study focuses on the 135 (31.3\%) patients who had LN-positive disease.
Regional lymphadenectomy was generally performed in patients with enlarged LNs on preoperative axial imaging or with adenopathy detected during surgery. The lymphadenectomy template was not standardized. Of the 135 patients with LNpositive disease, $119(88.1 \%)$ had open and 16 (11.9\%) laparoscopic RNU. Although not standardized due to the multi-institutional nature of the study, lymphadenectomy comprised the para-aortic, paracaval or interaortocaval nodes from the hilus to the inferior mesenteric artery for renal pelvic and proximally ureteric tumours, the renal hilus to the bifurcation of the common iliac artery for mid-ureteric tumours, and ipsilateral pelvic nodes for distal ureteric tumours. Overall, 57 patients $(43.2 \%)$ received adjuvant chemotherapy; none of the patients received neoadjuvant treatment.

The total number of identified LNs and the number of LNs involved with primary urothelial tumours were documented. All pathological slides were re-reviewed by genitourinary pathologists according to strictly defined criteria and blinded to the original pathology slides and clinical outcomes. The disease was staged pathologically according to the 2002 TNM classification of the American Joint Committee on Cancer. Tumours were graded according to the 1998 WHO/International Society of Urologic Pathology consensus classification [18]. Besides tumour staging and grading, the presence of significant tumour necrosis (threshold 10\%), lymphovascular invasion (LVI) and concomitant carcinoma in situ (CIS) were assessed in every representative section.

Patients were generally followed every 3-4 months for the first year, semi-annually from the second to the fifth, and annually thereafter. The follow-up comprised a physical examination, routine serum chemistry studies, radiographic evaluation of the contralateral remaining UT and the urinary bladder, and chest radiography. Elective bone scans and chest/abdominal/ pelvic CT or MRI were used when clinically indicated. The median (range) follow-up was $4.1(0.9-14.0)$ years.

The cause of death was determined by the treating physicians, by chart review corroborated by death certificates, or by death certificates alone. Perioperative mortality lany death within 30 days of surgery or before discharge) was censored at time of death for UC-specific survival analyses.

Univariable recurrence and survival probabilities after RNU were estimated using the Kaplan-Meier method. Univariable and multivariable Cox regression models addressed time to recurrence and diseasespecific mortality after RNU. In all models proportional hazards assumptions were systematically verified, using the GrambschTherneau residual-based test [19]. The change in predictive accuracy resulting from adding variables of interest to standard predictor variables was quantified with Harrell's concordance index $[20,21]$. A value of 1.0 indicates perfect predictions, while 0.5 is equivalent to random chance. Internal validation was performed using 200bootstrap re-samples $[20,21]$. Estimates of predictive accuracy were expressed as proportions and compared using the MantelHaenszel test. All reported $P$ values are twosided and statistical significance was set at $\leq 0.05$. Nominal $P$ values are given; no adjustments were made for the number of $P$ values calculated.

\section{RESULTS}

Table 1 summarizes the clinical and pathological characteristics of 135 patients with LN-positive UTUC. The proportion of patients with LVI among the LN-positive patients was significantly higher than in those with stage pNO (297) in which only 80 patients $(26.9 \%)$ presented with LVI $(P<0.001)$. When considering the dominant urothelial lesion (as determined by pathological size and T classification), $73 \%$ of tumours were in the renal pelvis and $27 \%$ in the ureter.

In a preliminary study we assessed the discriminative value of each LND threshold for prognosis. Kaplan-Meier analyses of serial increments of the percentage threshold showed that a threshold of $30 \%$ was the best discriminator for both cancer recurrence and survival (data not shown). Overall, 96 patients (71.1\%) had a LND of $\geq 30 \%$.

Within a median follow-up of 4.1 years, 90 patients (66.7\%) had disease recurrence and 76 (58\%) died from UTUC. The mean (SEM) 5and 10-year recurrence-free survival (RFS) probabilities were 27 (4)\% and 26 (4)\%, respectively; the 5 - and 10 -year DSS 
probabilities were $33(5) \%$ and 32 (5)\%, respectively. The median time to disease recurrence was 1 year and the median time to cancer-specific death 2.1 years. When compared with the 297 patients who had a lymphadenectomy and had pNO disease, patients with LN-positive disease had a significantly higher recurrence (hazard ratio 2.42, $P=0.001$ ) and disease-specific mortality $(2.44, P=0.004)$ rates after adjusting for the effects of tumour stage, grade and LVI.

Table 2 shows the univariable and multivariable Cox regression analyses predicting disease recurrence and diseasespecific death. A higher pathological tumour stage of the primary UTUC was associated with higher risk of disease recurrence (hazard ratio $3.85, P<0.001)$ and disease-specific mortality (4.26, $P<0.001)$. When analysed separately, neither the total number of LNs examined nor the total number of LNs involved with tumour were associated with disease recurrence $(P=0.73$ and 0.99 , respectively) or DSS ( $P=0.66$ and 0.43 , respectively).

Patients with a LND of $\geq 30 \%$ were at significantly greater risk of both disease recurrence, with 5 -year rates of 38 (8)\% vs $25(5) \%(P=0.021)$ and mortality, with 5-year rates of $48(9) \%$ vs $30(6) \%(P=0.032)$ than those with a LND of $<30 \%$ (Fig. 1). In multivariable analyses that adjusted for the effects of tumour stage, grade and LVI, LND coded as a continuous variables was not associated with cancer recurrence or DSS. However, using the threshold of 30\%, LND was independently associated with both disease recurrence and DSS $(P=0.005$ and 0.004 , respectively).

The accuracies for predicting tumour recurrence and DSS of the base model comprising pT stage, tumour grade and LVI were $65.2 \%$ and $64.9 \%$, respectively. Adding LND using the 30\% threshold significantly improved the predictive accuracies for both disease recurrence and DSS, to $69.8 \%$ and 68.1\% (both $P<0.001$ ).

Adjuvant chemotherapy (59 patients) was used in equal proportions in patients with a LND of $<30 \%$ (21 of 39 ) and $\geq 30 \%$ (38 of 96) $(P=0.19)$. Adjuvant chemotherapy had no effect on disease recurrence $(P=0.738)$ or DSS $(P=0.246)$. Analyses were re-run after stratifying by adjuvant therapy, which resulted in consistent patterns and $P$ values.

\begin{tabular}{|c|c|c|}
\hline Variable & Median (range) or $n(\%)$ & TABLE 1 \\
\hline Age, years & $68.0(27-90)$ & Descriptive characteristics \\
\hline \multicolumn{2}{|l|}{ Gender } & of 135 patients with LN \\
\hline Men & $88(65.2)$ & metastasis at RNU for UTUC \\
\hline Women & $47(34.8)$ & \\
\hline \multicolumn{3}{|l|}{ Pathological tumour stage } \\
\hline pTO & $1(0.7)$ & \\
\hline pT1 & $7(5.2)$ & \\
\hline pT2 & $21(15.6)$ & \\
\hline pT3 & $74(54.8)$ & \\
\hline pT4 & $32(23.7)$ & \\
\hline \multicolumn{3}{|l|}{ Pathological tumour grade } \\
\hline Low & $10(7.4)$ & \\
\hline High & $125(92.6)$ & \\
\hline Tumour necrosis & $75(55.6)$ & \\
\hline Concomitant CIS & $58(43.0)$ & \\
\hline LVI & $97(71.9)$ & \\
\hline \multicolumn{3}{|l|}{ No. of LNs examined } \\
\hline all patients & $4(1-41)$ & \\
\hline LND $<30 \%$ & $10(3-41)$ & \\
\hline LND $\geq 30 \%$ & $2(1-29)$ & \\
\hline \multicolumn{3}{|c|}{ No of LNs involved with cancer } \\
\hline All patients & $2(1-22)$ & \\
\hline LND $<30 \%$ & $1(1-8)$ & \\
\hline LND $\geq 30 \%$ & $2(1-22)$ & \\
\hline LND, \% & $50(3-100)$ & \\
\hline No. of patients LND $\geq 30 \%$ & $96(71.1)$ & \\
\hline
\end{tabular}

TABLE 2 Univariable and multivariable Cox regression analyses predicting disease relapse and DSS in 135 patients with LN metastasis at RNU for UTUC

\begin{tabular}{llc} 
& Hazard ratio; $P$ & \\
\cline { 2 - 3 } Variable & Univariable & Multivariable \\
\hline Disease relapse & & $<0.001$ \\
pT stage & $<0.001$ & $1.33 ; 0.35$ \\
$\quad$ pT3 vs $\leq$ pT2 & $1.52 ; 0.08$ & $3.85 ;<0.001$ \\
pT4 vs $\leq$ pT2 & $4.78 ;<0.001$ & $2.88 ; 0.09$ \\
Tumour grade (high vs low) & $4.10 ; 0.02$ & $0.97 ; 0.57$ \\
LVI & $1.16 ; 0.13$ & $1.84 ; 0.05$ \\
LND (threshold 30\%) & $2.61 ; 0.03$ & \\
Predictive accuracy, \% & & \\
$\quad$ base model (pT stage, grade and LVI & 65.2 & \\
$\quad$ base model + LND 30\% & 69.8 & \\
$\quad$ gain with LND 30\% & $+4.6 ;<0.001$ & $1.53 ; 0.22$ \\
DSS & & $4.26 ;<0.001$ \\
pT stage & $<0.001$ & $2.84 ; 0.16$ \\
$\quad$ TT3 vs $\leq \mathrm{pT} 2$ & $1.70 ; 0.10$ & $0.98 ; 0.17$ \\
pT4 vs $\leq \mathrm{pT} 2$ & $4.78 ;<0.001$ & $1.73 ; 0.04$ \\
Tumour grade (high vs low) & $4.93 ; 0.03$ & \\
LVI & $1.23 ; 0.11$ & \\
LND (threshold 30\%) & $2.31 ; 0.03$ & \\
Predictive accuracy, \% & & \\
$\quad$ base model (pT stage, grade and LVI)\% & 64.9 & \\
base model + LND 30\% & 68.1 & \\
gain with LND 30\% & $+3.2 ;<0.001$ &
\end{tabular}


FIG. 1. Kaplan-Meier estimates for RFS (A) and DSS (B) in $135 \mathrm{LN}$-positive patients after RNU when stratified by a LND threshold of 30\%. Patients with a LND of $<30 \%$ (blue curve; 39 ) were at significantly lower risk of disease recurrence and disease-specific death than patients with a LND of $\geq 30 \%$ (green curve; 96) ( $\mathrm{P}=0.021$ and 0.032 , respectively). The boxes include the number of patients still at risk after 12, 24, 60 and 96 months.
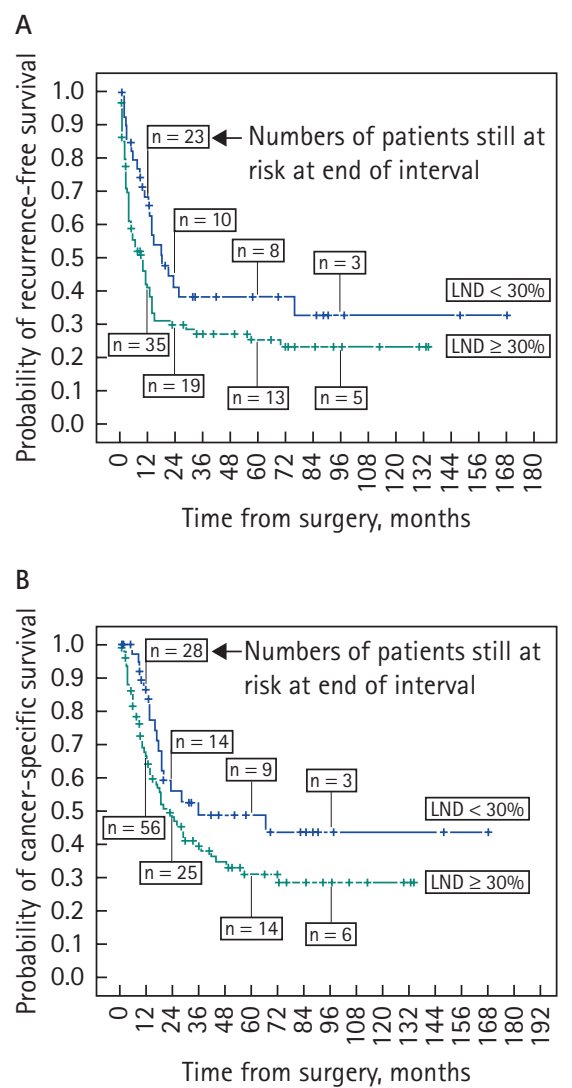

\section{DISCUSSION}

We evaluated the LND concept for the first time in UTUC and found that LND using a threshold of $30 \%$ was a powerful predictor of disease recurrence and DSS in patients with LN-positive UTUC after RNU. Of patients with a LND of $<30 \%, 25$ and $30 \%$ had cancer recurrence and died within 5 years of RNU, respectively, compared to $38 \%$ and $48 \%$ with a LND of $\geq 30 \%$, respectively. In multivariable analyses that adjusted for the effects of standard pathological features (i.e. pathological stage, tumour grade and LVI), patients with a LND of $\geq 30 \%$ had 1.8 times the risk of disease recurrence and 1.7 times the risk of disease-specific death after RNU than patients with a LND of $<30 \%$. Moreover, adding LND using a threshold of 30\% improved the predictive accuracies of the base models including standard pathological features for predicting both disease recurrence and DSS, by a prognostic and clinically significant margin.

The overall incidence of $L N$-positive disease at RNU in the present series was 31\% (135 of 432 patients) which is within the range of previous reports $[5,6]$. Nodal metastasis at RNU portends a poor prognosis, with survival rates in earlier series as low as $\approx 10 \%$ [7]. In bladder cancer, recent series show improved survival in patients who have positive $L N \mathrm{Ns}_{\text {, }}$ with 5-year overall and RFS rates of 20-30\%, possibly due to more complete LN dissection $[11,22]$.

Recently, increasingly many reports show advantages for the concept of LND compared to or in addition to the absolute number of involved LNs in predicting RFS of patients with LN-positive cancer $[14,16,17]$. This concept incorporates the tumour burden and the extent of LN dissection. To our knowledge, the LND concept has never been evaluated in UTUC. In the present study we tested the predictive value of LND in a large multicentre retrospective dataset of patients with $L N$ positive UTUC. LND and pathological tumour stage were the only features independently associated with disease recurrence and survival in patients with LN-positive UTUC. In addition, similar to a previous study in bladder UC, we found that LND was a more powerful predictor of RFS than the absolute numbers of involved LNs [14]. Only the combination of the number of involved LNs and the number of examined LNs was statistically significant for outcomes. This could be explained because the LND takes into account the potential therapeutic value of a locoregional LN dissection. This finding is of potential clinical significance, as LND is a variable that can, at least in theory, be influenced by the surgeon through an extended lymphadenectomy. Furthermore, LND remained a significant predictor of clinical outcomes after adjusting for the effect of adjuvant chemotherapy. We identified 30\% as the best LND threshold for stratifying patients into significantly different risk groups. This threshold was established after systematically assessing the association of $10 \%$ increments of LND with clinical outcomes after RNU. While all patients with LN-positive disease have a poor prognosis, patients with a LND of $\geq 30 \%$, regardless of pathological features, have significantly worse survival rates than those with a LND of $<30 \%$, and therefore might benefit from early aggressive therapy.

There are several limitations to the present study. Although we used many internal and external reviews of the dataset, we excluded from this analysis patients for whom we could not obtain complete information, possibly creating a selection bias. Additional biases include differences in the performance of the Iymphadenectomy, and the extent and location of the lymphadenectomy, by study site and individual surgeon. As to the extent of lymphadenectomy in the present series, our model of LND is based on relatively few LNs removed during RNU. Thus, the reliability of a threshold of $30 \%$ can be questioned. Furthermore, for the number of evaluated $\mathrm{LNs}$, there is a discrepancy between the group of patients with a LND of $<30 \%$ (median 10 LNs) and those with a LND of $>30 \%$ (median two LNs). This might reflect a bias, but patients with a LND of $<30 \%$ have obviously had a more extended lymphadenectomy, which contributed to a lower LND value and translated into a better outcome in the present study. However, that neither the total number of LNs examined nor the total number of LNs involved with cancer were associated with disease recurrence or DSS suggests that the extent of lymphadenectomy was insufficient in most patients.

Finally, review and interpretation by many genitourinary pathologists can potentially introduce a diagnostic bias. While these biases cannot be controlled for in the analysis, the multi-institutional nature of our study and the use of 'local' pathological interpretation might make our conclusions more relevant and applicable in both the academic and community settings, reflecting a more realistic picture of the management of UTUC.

Nonetheless, even with these limitations and being aware of the fact that LND as a ratio must be interpreted with caution, our results suggest that the concept of LND is relevant in patients with UTUC who are treated with RNU, even after adjusting for adjuvant chemotherapy.

We conclude that LND is an independent predictor of clinical outcomes in patients with UTUC treated with RNU. Our findings also support recent evidence that lymphadenectomy performed in conjunction with RNU has a therapeutic role in LN- 
positive patients with UTUC. Thus we furthermore argue in favour of extended lymphadenectomy in these patients, as it decreases the LND ratio. LND offers an attractive proxy measure of quality of care and might be useful in future staging systems. Moreover, future adjuvant therapies and clinical trials should consider applying the LND concept, as it might help to reduce the surgical bias and the bias in the extent of lymphadenectomy, both of which are currently not standardized. Our preliminary data support systematic nodal ratio analysis from multi-institutional patient datasets. A prospective validation of LND and standardized lymphadenectomy during RNU are required.

\section{CONFLICT OF INTEREST}

None declared.

\section{REFERENCES}

1 Oosterlinck W, Solsona E, van der Meijden AP et al. EAU guidelines on diagnosis and treatment of upper urinary tract transitional cell carcinoma. Eur Urol 2004; 46: 147-54

2 Zigeuner R, Pummer K. Urothelial carcinoma of the upper urinary tract: surgical approach and prognostic factors. Eur Urol 2008; 53: 720-31

3 Lehmann J, Suttmann H, Kovac I et al. Transitional cell carcinoma of the ureter: prognostic factors influencing progression and survival. Eur Urol 2007; 51: 1281-8

4 Roscigno M, Cozzarini C, Bertini R et al. Prognostic value of lymph node dissection in patients with muscleinvasive transitional cell carcinoma of the upper urinary tract. Eur Urol 2008; 53 : 794-802

5 Kondo T, Nakazawa H, Ito F, Hashimoto Y, Toma H, Tanabe K. Primary site and incidence of lymph node metastases in urothelial carcinoma of upper urinary tract. Urology 2007; 69: 265-9

6 Brausi MA, Gavioli M, De Luca G et al. Retroperitoneal lymph node dissection (RPLD) in conjunction with nephroureterectomy in the treatment of infiltrative transitional cell carcinoma (TCC) of the upper urinary tract: impact on survival. Eur Urol 2007; 52: 1414-8

7 Novara G, De Marco V, Gottardo F et al. Independent predictors of cancer-specific survival in transitional cell carcinoma of the upper urinary tract: multiinstitutional dataset from 3 European centers. Cancer 2007; 110: 1715-22

8 Ozsahin M, Zouhair A, Villa S et al. Prognostic factors in urothelial renal pelvis and ureter tumours: a multicentre Rare Cancer Network study. Eur J Cancer 1999; 35: 738-43

9 Park J, Park S, Song C et al. Effectiveness of adjuvant chemotherapy in transitional cell carcinoma of the urinary bladder with lymph node involvement and/or lymphovascular invasion treated by radical cystectomy. Urology 2007; 70 : 257-62

10 Secin FP, Koppie TM, Salamanca Jl et al. Evaluation of regional lymph node dissection in patients with upper urinary tract urothelial cancer. Int J Uro/ 2007; 14: 26-32

11 Shariat SF, Karakiewicz PI, Palapattu GS et al. Outcomes of radical cystectomy for transitional cell carcinoma of the bladder: a contemporary series from the Bladder Cancer Research Consortium. J Urol 2006; 176: 2414-22

12 Herr HW, Bochner BH, Dalbagni G, Donat SM, Reuter VE, Bajorin DF. Impact of the number of lymph nodes retrieved on outcome in patients with muscle invasive bladder cancer. J Urol 2002; 167: 1295-8

13 Stein JP, Penson DF, Cai J et al. Radical cystectomy with extended lymphadenectomy: evaluating separate package versus en bloc submission for node positive bladder cancer. J Uro/ 2007; 177: 876-81

14 Stein JP, Cai J, Groshen S, Skinner DG. Risk factors for patients with pelvic lymph node metastases following radical cystectomy with en bloc pelvic lymphadenectomy: concept of lymph node density. J Urol 2003; 170: 35-41

15 Kondo T, Nakazawa H, Ito F, Hashimoto Y, Toma H, Tanabe K. Impact of the extent of regional lymphadenectomy on the survival of patients with urothelial carcinoma of the upper urinary tract. J Urol 2007; 178: 1212-7

16 Daneshmand S, Quek ML, Stein JP et al. Prognosis of patients with lymph node positive prostate cancer following radical prostatectomy: long-term results. J Urol 2004; 172: 2252-5

17 Ooki A, Yamashita K, Kobayashi N et al. Lymph node metastasis density and growth pattern as independent prognostic factors in advanced esophageal squamous cell carcinoma. World J Surg 2007; 31: 2184-91

18 Epstein Jl, Amin MB, Reuter VR, Mostofi FK. The World Health Organization/International Society of Urological Pathology consensus classification of urothelial (transitional cell) neoplasms of the urinary bladder. Bladder Consensus Conference Committee. Am J Surg Pathol 1998; 22: 1435-48

19 Grambsch PM, Therneau TM. Proportional hazards tests and diagnostics based on weighted residuals. Biometrika 1994; 81: 515-9

20 Harrell FE Jr, Califf RM, Pryor DB, Lee $\mathrm{KL}$, Rosati RA. Evaluating the yield of medical tests. JAMA 1982; 247: 2543-6

21 Harrell FE Jr, Lee KL, Mark DB. Multivariable prognostic models: issues in developing models, evaluating assumptions and adequacy, and measuring and reducing errors. Stat Med 1996; 15: 361-87

22 Stein JP, Lieskovsky G, Cote R et al. Radical cystectomy in the treatment of invasive bladder cancer: long-term results in 1,054 patients. J Clin Oncol 2001; 19: 666-75

Correspondence: Shahrokh F. Shariat, Memorial Sloan-Kettering Cancer Center, 1275 York Avenue, Box 27, New York, NY 10065, USA.

e-mail: sfshariat@gmail.com or shariats@mskcc.org

Abbreviations: RNU, radical nephroureterectomy; UTUC, upper tract urothelial carcinoma; LN(D), lymph node (density); DSS, disease-specific survival; LVI, lymphovascular invasion; CIS, carcinoma in situ; RFS, recurrence-free survival. 